\title{
A Novel Convenient Synthesis of Pyridinyl and Quinolinyl Triflates and Tosylates via One-Pot Diazotization of Aminopyridines and Aminoquinolines in Solution
}

Assiya Zh. Kassanova

Elena A. Krasnokutskaya*

Perizat S. Beisembai

Victor D. Filimonov*

Synthesis 2016, 48, 256.

For the final online and print versions, the Acknowledgement section was updated to indicate which work was supported by the Russian Scientific Fund. 